\title{
MicroRNA Profiling Response to Acupuncture Therapy in Spontaneously Hypertensive Rats
}

\author{
Jia-You Wang, ${ }^{1,2}$ Hui Li, ${ }^{2}$ Chun-Mei Ma, ${ }^{1}$ Jia-Lu Wang, \\ Xin-Sheng Lai, ${ }^{3}$ and Shu-Feng Zhou ${ }^{2,4}$ \\ ${ }^{1}$ Department of Human Anatomy, College of Fundamental Medical Sciences, Guangzhou University of Chinese Medicine, \\ Guangzhou, Guangdong 510006, China \\ ${ }^{2}$ Department of Pharmaceutical Sciences, College of Pharmacy, University of South Florida, 12901 Bruce B. Downs Boulevard, \\ MDC 30, Tampa, FL 33612, USA \\ ${ }^{3}$ Department of Acupuncture and Moxibustion, College of Acupuncture and Moxibustion, Guangzhou University of Chinese Medicine, \\ Guangzhou, Guangdong 510006, China \\ ${ }^{4}$ Guizhou Provincial Key Laboratory for Regenerative Medicine, Stem Cell and Tissue Engineering Research Center \& Sino-US Joint \\ Laboratory for Medical Sciences, Guiyang Medical University, Guiyang, Guizhou 550004, China
}

Correspondence should be addressed to Xin-Sheng Lai; lai1023@163.com and Shu-Feng Zhou; szhou@health.usf.edu

Received 19 May 2014; Revised 11 July 2014; Accepted 12 July 2014

Academic Editor: William C. Cho

Copyright (C) 2015 Jia-You Wang et al. This is an open access article distributed under the Creative Commons Attribution License, which permits unrestricted use, distribution, and reproduction in any medium, provided the original work is properly cited.

\begin{abstract}
MicroRNAs (miRNAs) are a group of endogenous noncoding RNAs that play important roles in many biological processes. This study aimed to check if miRNAs were involved in the response to acupuncture in rats. Microarray analysis was performed to compare the miRNA expression profiles of medulla in spontaneously hypertensive rats (SHRs) treated with or without acupuncture. Our microarray analysis identified 222 differentially expressed miRNAs in the medulla of SHRs treated with acupuncture at taichong acupoint. Among these miRNAs, 23 miRNAs with a significant difference were found in acupuncture-treated SHRs compared to untreated rats. These 23 miRNAs could regulate 2963 target genes which were enriched in at least 14 pathways based on our bioinformatic analysis. miRNA-339, miR-223, and miR-145 were downregulated in the medulla of SHRs compared to normotensive rats. Notably, these miRNAs were upregulated to basal levels in the medulla of SHRs treated with acupuncture at taichong in comparison with SHRs receiving acupuncture at nonacupoint group or SHRs without any treatment. Our findings have revealed significant changes of a panel of selective miRNAs in hypertensive rats treated at taichong acupoint. These data provide insights into how acupuncture elicits beneficial effects on hypertension.
\end{abstract}

\section{Introduction}

Hypertension, a major risk factor for cardiovascular disease, affects approximately one billion individuals worldwide [1], including 78 million (33\%) American adults $\geq 20$ years of age (i.e., it affects one in three adults in the United States) [2]. Although a number of treatment strategies have been developed for this disease, only $75 \%$ of hypertensive patients received pharmacologic treatment, and only $53 \%$ of those with documented hypertension have their condition controlled to target levels [2]. The major barriers to successful pharmacotherapeutic control of hypertension are poor drug response, adverse side effects, and low patient compliance [3].
Acupuncture originated in ancient China at least 2,500 years ago. Although there is some controversy in mainstream Western medicine, it has become one of the most widely practiced forms of alternative medicine in the world [4]. For example, estimated 3 million American adults receive acupuncture treatment each year [5]. The US Internal Revenue Service approved acupuncture as deductible medical expense in 1973. The National Institute of Health has sponsored a Consensus Development Conference on Acupuncture in 1997 [6]. WHO has approved acupuncture as an alternative therapy for the treatment of at least 64 types of diseases. On November 16, 2010, acupuncture has been listed 
by the United Nations Educational, Scientific, and Cultural Organization as "Intangible Cultural Heritage."

Since the 1970s, a number of animal and clinical studies have demonstrated the effectiveness of acupuncture at specific acupoints to lower blood pressure in essential hypertension [7-10]. Unfortunately, the underlying mechanisms through which acupuncture lowers blood pressure remain unclear. Studies have suggested the involvement of nitric oxide (NO) [11], neurotransmitters [12], aldosterone, endothelin, and angiotensin II [13, 14], and acetylcholine, opioids, $\gamma$-aminobutyric acid, serotonin, and endocannabinoids in the brain all appear to contribute to the acupuncture's antihypertensive effect $[15,16]$.

The small noncoding microRNAs (miRNAs) have critical functions in the regulation of various critical biological processes such as cell metabolism, proliferation, death, and development $[17,18]$. To date, there are more than 2,000 miRNAs reported in humans. A number of studies have found that miRNAs respond to various therapeutic interventions such as pharmacotherapy, physical therapy, and radiotherapy. However, there is no information on the effect of acupuncture therapy on miRNA profiles in animals and humans. The objective of the present study was to determine whether miRNAs were involved in the response to acupuncture therapy in rats. This study is the first to examine the miRNA response in the medulla of rats to acupuncture at the taichong (LR3) point compared to stimulation at a nonacupoint in spontaneously hypertensive rats (SHRs).

\section{Materials and Methods}

2.1. Ethics Statement. All animal experiments were performed at the Laboratory Animal Center of Guangzhou University of Chinese Medicine, Guangzhou, China. The procedure was approved by the Ethics Committee of Guangzhou University of Chinese Medicine, Guangzhou, China [permit number: SYXK (Yue) 2008-0085].

2.2. Chemicals and Reagents. The 6th generation of miRCURYTM LNA Array (v.16.0) was obtained from KangChen Bio-tech (Shanghai, China), which contains more than 1891 capture probes, covering all human, mouse, and rat microRNAs annotated in miRBase 16.0 , as well as all viral microRNAs related to these species. In addition, this array contains capture probes for 66 new miRPlus human microRNAs. All chemicals and reagents used were of analytical grade.

2.3. Animals. The experiments were performed with SHRs and Sprague-Dawley (SD) rats provided by Beijing Vital River Laboratory Animals Co., Ltd (Beijing, China). The rats were housed in cages maintained in a temperature- and humidity-controlled room at the Laboratory Animal Center of Guangzhou University of Chinese Medicine, Guangzhou, China. The animals were given a standard diet. The SHRs with confirmed blood pressure $\geq 140 \mathrm{mmHg}$ were included and randomly divided into three groups: (1) acupuncture at taichong group $(n=6)$ treated with acupuncture at LR3 point, (2) acupuncture at nonacupoint group $(n=6)$ treated with acupuncture at nonacupoints, and (3) model group ( $n=$ 6) untreated with acupuncture throughout the duration of the experiment. In the literature, SD rats have been used as the normotensive controls in comparison to SHRs $[19,20]$ and thus they were used as normal control group $(n=6)$ in this study.

2.4. Acupuncture Procedure. A number of clinical and animal studies have reported the efficacy of acupuncture at taichong point in reducing hypertension [21-23]. The acupuncture treatment procedure was the same as described previously by us [22]. Briefly, in acupuncture taichong group, acupuncture was performed at bilateral taichong points (LR3) located between the 1st and the 2nd metatarsal of dorsal foot, while, in acupuncture nonacupoint group, acupuncture was done at bilateral nonacupoint located at the fossa between the 3rd and 4th metatarsal of dorsal foot (Figure 1). As previously described $[22,24]$, the rats were lightly immobilized and the acupuncture needles were inserted to a depth of $3 \mathrm{~mm}$ at the appropriate location bilaterally, twisted at a rate of 80 spins per min, and removed afterward. This treatment was given daily (treatment lasting $5 \mathrm{~min} /$ day) for 7 consecutive days. After the last acupuncture treatment, rats were anesthetized and their medullas were quickly dissected. The medullas were preserved in liquid nitrogen until analysis.

2.5. Microarray Analysis. Total RNA was isolated using TRIzol (Invitrogen, Grand Island, NY, USA) and miRNeasy minikit (QIAGEN) according to manufacturer's instruction. After RNA isolation from the samples, the miRCURY Hy3/Hy5 Power labeling kit (Exiqon, Vedbaek, Denmark) was used according to the manufacturer's guideline for miRNA labelling. One microgram of each sample was $3^{\prime}$ end-labeled with Hy3 fluorescent label, using T4 RNA ligase. After stopping the labeling procedure, the Hy3-labeled samples were hybridized on the miRCURY LNA Array (v.16.0) (Exiqon) according to the manufacturer's manual. The slides were scanned using the Axon GenePix 4000B microarray scanner (Axon Instruments, Foster City, CA, USA). Scanned images were then imported into GenePix Pro 6.0 software (Axon) for grid alignment and data extraction. Replicated miRNAs were averaged and miRNAs in which intensities $>50$ in all samples were chosen for calculating the normalization factor. The data were normalized using the Median normalization method and after normalization differentially expressed miRNAs were identified through Volcano Plot filtering. In addition, hierarchical clustering was performed using MEV software (v4.6, TIGR).

2.6. Bioinformatics Studies. Computational target prediction of miRNAs was conducted by miRDB online searching program (http://mirdb.org/miRDB/). The enriched KEGG pathway for these targets was analyzed in DAVID Bioinformatics Resources 6.7 (http://david.abcc.ncifcrf.gov/).

2.7. Quantitative Real-Time PCR ( $q R T-P C R)$. The rat medulla was homogenized in $400 \mu \mathrm{L}$ of TRIzol reagent (Invitrogen, Grand Island, NY, USA). To avoid contamination with 


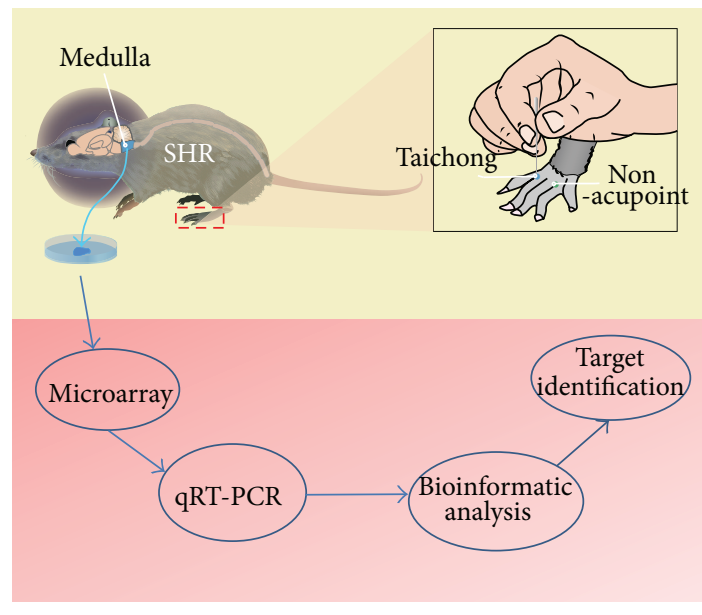

FIGURE 1: The acupuncture procedure at taichong point and nonacupoint in SHRs. In taichong group of the SHRs, acupuncture was performed at bilateral LR3 located between the 1st and the 2nd metatarsal of dorsal foot, while, in nonacupoint group, acupuncture was done at bilateral nonacupoint located at fossa between the 3rd and 4 th metatarsal of dorsal foot.

genomic DNA, the RNA samples were treated with RNasefree DNase (Promega, Madison, WI, USA). Reverse transcription was performed using M-MLV reverse transcriptase (Promega, Madison, WI, USA). Real-time RT-PCRs were carried out using an ABI PRISM 7500 Sequence Detection System (Applied Biosystems, Carlsbad, CA, USA) and the SYBR Green PCR Master Mix kit (Toyobo Co., Ltd., Osaka, Japan). The $2^{-\Delta \Delta C t}$ method was used to analyze the RT-PCR data and the ${ }^{\Delta \mathrm{Ct}}$ of each miRNA was determined relative to U6 RNA and endogenous control miRNA that is robustly and invariantly expressed in all cell types. The experiments were performed in triplicate, and consistent results were obtained.

2.8. Statistical Analysis. Data are presented as the mean \pm SD. Multiple comparisons were evaluated by one-way analysis of variance (ANOVA) followed by Tukey's multiple comparison procedure, with $P<0.05$ considered significant. All statistical tests were performed using Prism software version 6.0 (GraphPad Software Inc., Chicago, IL, USA).

\section{Results}

3.1. miRNAs Profiling Response to Acupuncture Therapy in SHRs. To reveal miRNAs profiling response to acupuncture therapy in SHRs, microarrays containing 1891 human, rat, and mouse miRNAs were used to examine the expression of miRNAs in medullas after acupuncture treatment at taichong point in SHRs. The analyses were performed using total RNA collected from three rat medullas. Statistical analysis of the miRNA expression data identified 222 miRNAs with significant changes in the expression, with 23 miRNAs having expression levels greater or equal to a 1.5 -fold change in SHRs with or without acupuncture treatment (Tables 1 and 2). Among these 23 differentially expressed miRNAs, 8 of 23 miRNAs were significantly upregulated, while 15 of 23
TABLE 1: miRNAs upregulated by acupuncture in the medulla of SHRs.

\begin{tabular}{lccc}
\hline $\begin{array}{l}\text { Mature miRNAs } \\
\text { (rno-miR-) }\end{array}$ & $\begin{array}{c}\text { Fold-change } \\
(\text { mean } \pm \text { SEM) }\end{array}$ & $P$ value & $\begin{array}{c}\text { miRBase accession } \\
\text { number }\end{array}$ \\
\hline 339 & 2.13 & 0.0214 & MIMAT0000583 \\
223 & 3.34 & 0.0011 & MI0000963 \\
145 & 3.02 & 0.0059 & MI0000918 \\
451 & 3.32 & 0.0097 & MI0001731 \\
193 & 2.88 & 0.0300 & MI0000936 \\
378 & 2.30 & 0.0135 & MI0003719 \\
423 & 1.97 & 0.0277 & MI0006145 \\
let-7b & 1.70 & 0.0443 & MIMAT0004705 \\
\hline
\end{tabular}

Each experiment was performed in triplicate. The fold change is presented as the mean \pm SEM. ${ }^{*}$ Indicates antisense mature miRNA.

TABLE 2: miRNAs downregulated by acupuncture in the medulla of SHRs.

\begin{tabular}{lccc}
\hline $\begin{array}{l}\text { Mature miRNAs } \\
\text { (rno-miR-) }\end{array}$ & $\begin{array}{c}\text { Fold-change } \\
(\text { mean } \pm \text { SEM) }\end{array}$ & $P$ value & $\begin{array}{c}\text { miRBase accession } \\
\text { number }\end{array}$ \\
\hline $7 \mathrm{a}$ & 0.52 & 0.0254 & MI0000641 \\
9 & 0.48 & 0.0491 & MI0000838 \\
128 & 0.45 & 0.0229 & MI0000900 \\
132 & 0.42 & 0.031 & MI0000905 \\
134 & 0.59 & 0.0037 & MI0000907 \\
182 & 0.24 & 0.0093 & MI0006133 \\
335 & 0.6 & 0.0475 & MI0000612 \\
382 & 0.44 & 0.0381 & MI0003548 \\
383 & 0.51 & 0.0324 & MI0003478 \\
434 & 0.44 & 0.0155 & MI0006147 \\
496 & 0.59 & 0.011 & MI0012622 \\
$135 \mathrm{a}$ & 0.45 & 0.0414 & MI0000908 \\
$136^{*}$ & 0.58 & 0.0131 & MIMAT0004733 \\
$376 \mathrm{~b}-5 \mathrm{p}$ & 0.54 & 0.0052 & MIMAT0003195 \\
$384-3 \mathrm{p}$ & 0.57 & 0.0225 & MIMAT0005310 \\
\hline
\end{tabular}

Each experiment was performed in triplicate. The fold change is presented as the mean \pm SEM. ${ }^{*}$ Indicates antisense mature miRNA.

miRNAs were significantly downregulated after acupuncture treatment.

3.2. Confirmation of Microarray Profiling Data by $q R T-P C R$. To validate the microarray profiling data, qRT-PCR was used to confirm the upregulated miRNAs including miRNA-339, miR-223, miR-145, and miR-451. The data showed that miR339 , miR-223, and miR-145 were significantly upregulated in medullas of SHRs treated with acupuncture at taichong point in contrast to the model group untreated with acupuncture (Figure 2). While being compared to the normal control group (healthy SD rats), miR-339, miR-223, and miR-145 were significantly downregulated in model group. There was no significant increase in miR-451 between acupuncture group and model group or normal group (data not shown). 


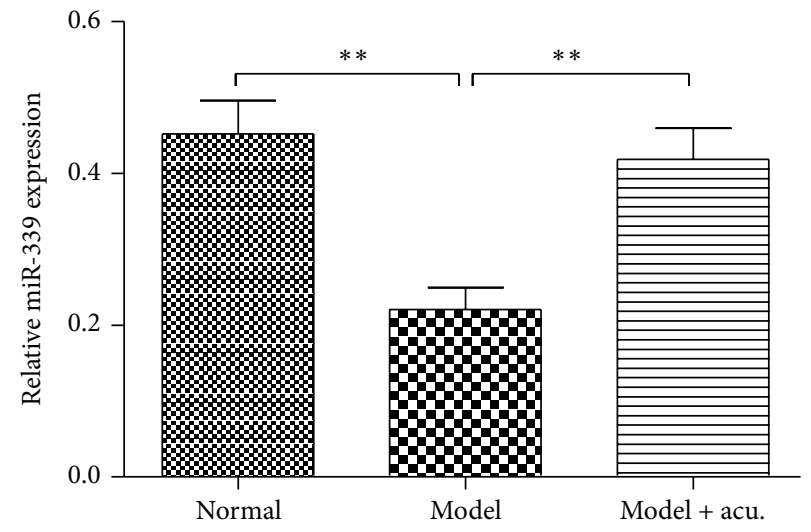

(a)

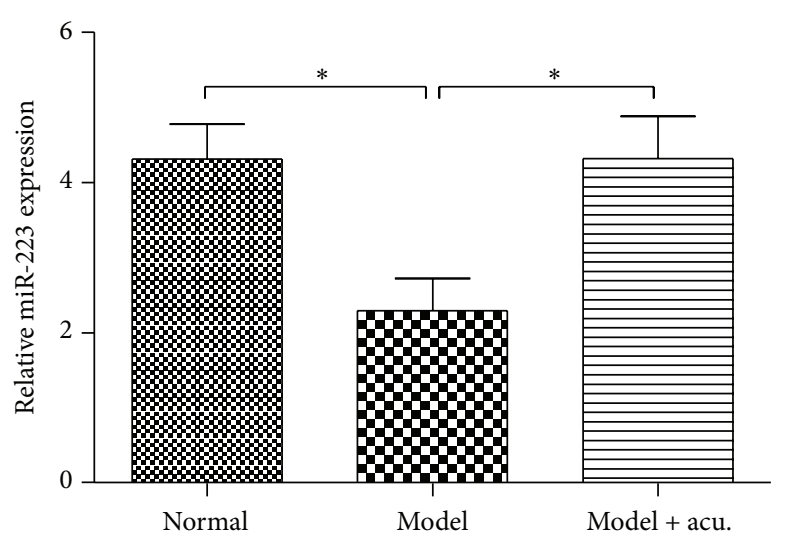

(b)

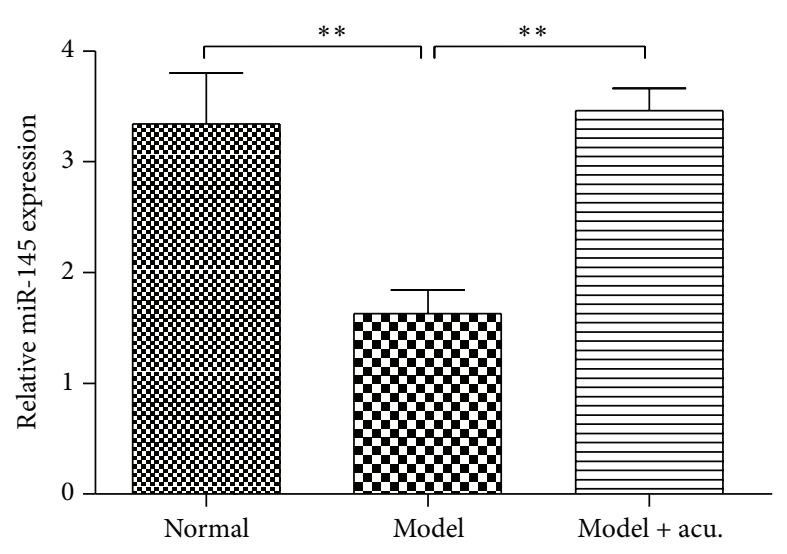

(c)

Figure 2: Confirmation of miR-339 (a), miR-223 (b), and miR-145 (c) expression changes in the medulla of SHRs treated with acupuncture at taichong point (model + acu.). The expression level of miR-339, miR-223, and miR-145 in rats was detected using qRT-PCR. The relative expression of these miRNAs was calculated in relation to levels of U6 RNA using the $2^{-\Delta \Delta \mathrm{Ct}}$ method. The result was repeated in triplicate independent experiments. ${ }^{*} P<0.05$; $^{* *} P<0.001$.

3.3. Acupuncture Treatment at Taichong Acupoint Elicits Specific miRNA Expression Changes in SHRs. Next, we compared the effect of acupuncture at taichong acupoint or at nonacupoint on miRNA expression changes in SHR medulla. The data showed that acupuncture at taichong point significantly increased miRNA-339, miR-223, and miR-145 levels in medullas of SHRs in contrast to the model group, while acupuncture at nonacupoint did not significantly alter miR339, miR-223, and miR-145 levels compared to the model group. Moreover, compared to acupuncture at nonacupoint, acupuncture at taichong point significantly upregulated the expression of miRNA-339, miR-223, and miR-145 in medullas of SHRs (Figure 3). These data showed significantly different miRNA response to real and sham acupuncture treatments in rats.

3.4. Possible Targets and Their Enriched Pathway of Acupuncture-Regulated miRNAs by Bioinformatics Analysis. To explore possible targets and signaling pathways involved in response to acupuncture, we used miRDB to predict the targets of the differentially expressed microRNAs responsive to acupuncture and used DAVID Bioinformatics
Resources 6.7 (http://david.abcc.ncifcrf.gov/) to analyze the enriched pathways. From the miRDB online database (http://mirdb.org/miRDB/), we identified 5223 targets for all 23 acupuncture-regulated miRNAs when the target score was set to $\geq 50$. When the target score cut-off was set at $\geq 60,2963$ possible targets were predicted. We further analyzed the enriched KEGG pathways for these 2963 targets and found 14 pathways, including neurotrophin signaling pathway, pathways in cancer, MAPK signaling pathway, Wnt signaling pathway, Ubiquitin mediated proteolysis, cell cycle, chemokine signaling pathway, oocyte meiosis, tight junction, $\mathrm{T}$ cell receptor signaling pathway, axon guidance, and TGF- $\beta$ signaling pathway (Table 3 ).

\section{Discussion}

In this study, we first found that miRNAs responded to acupuncture treatment in SHRs. Previous studies have demonstrated that physical therapy elicited remarkable miRNA profiling changes in humans [25-28] and mice [29]. Similarly, we have revealed that 222 medullar miRNAs were involved in acupuncture therapy in SHRs. Of these miRNAs, 


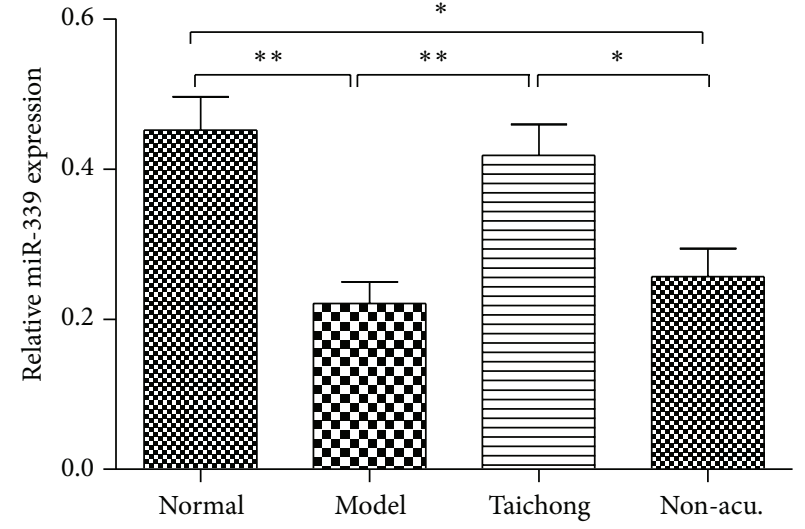

(a)

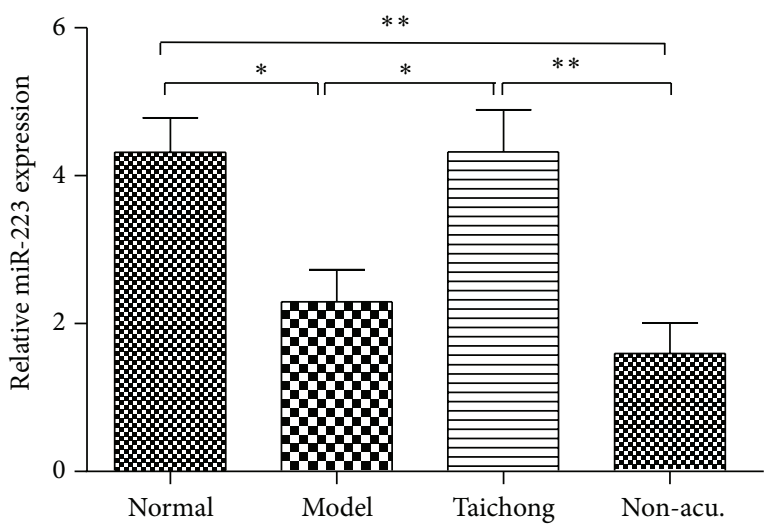

(b)

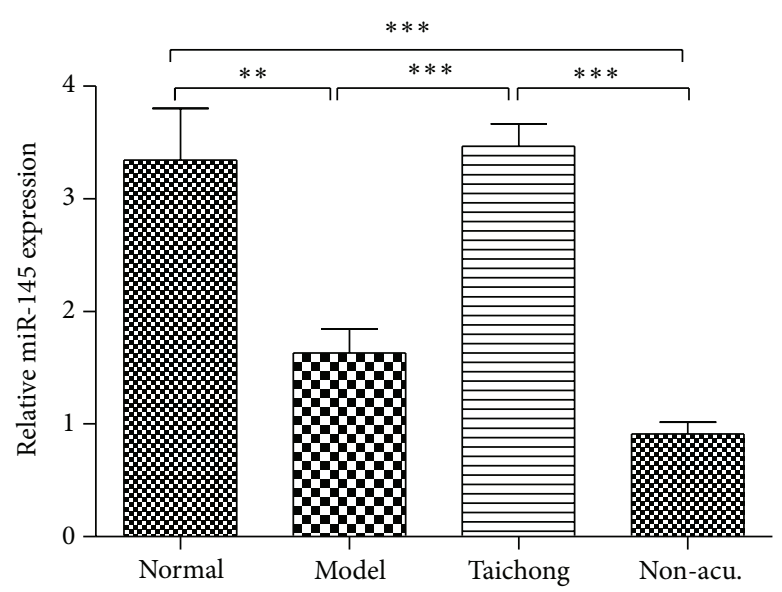

(c)

FIGURE 3: Acupuncture treatment at taichong acupoint elicits specific miRNA expression changes in SHRs. Acupuncture at taichong point (Taichong) significantly increased miR-339 (a), miR-223 (b), and miR-145 (c) expression levels in the medullas of SHRs compared to SHRs with nonacupoint treatment (non-acu.). ${ }^{*} P<0.05 ;{ }^{* *} P<0.001,{ }^{* * *} P<0.001$.

TABLE 3: The enriched KEGG pathways of acupuncture-responsive miRNAs in SHRs.

\begin{tabular}{|c|c|c|c|c|c|}
\hline Category & Term & Count & $\%$ & $P$ value & Benjamini \\
\hline KEGG_PATHWAY & Neurotrophin signaling pathway & 21 & 0.1 & $6.60 E-02$ & $6.50 E-01$ \\
\hline KEGG_PATHWAY & Pathways in cancer & 51 & 0.2 & $6.50 E-03$ & $1.60 E-01$ \\
\hline KEGG_PATHWAY & MAPK signaling pathway & 42 & 0.2 & $1.90 E-02$ & $3.20 E-01$ \\
\hline KEGG_PATHWAY & Wnt signaling pathway & 38 & 0.2 & $7.80 E-07$ & $1.40 E-04$ \\
\hline KEGG_PATHWAY & Ubiquitin mediated proteolysis & 31 & 0.1 & $3.70 E-05$ & $3.40 E-03$ \\
\hline KEGG_PATHWAY & Cell cycle & 29 & 0.1 & $2.40 E-04$ & $8.80 E-03$ \\
\hline KEGG_PATHWAY & Chemokine signaling pathway & 29 & 0.1 & $2.40 E-02$ & $3.50 E-01$ \\
\hline KEGG_PATHWAY & Calcium signaling pathway & 28 & 0.1 & $7.50 E-02$ & $6.40 E-01$ \\
\hline KEGG_PATHWAY & Oocyte meiosis & 27 & 0.1 & $1.60 E-04$ & $7.40 E-03$ \\
\hline KEGG_PATHWAY & Tight junction & 22 & 0.1 & $5.20 E-02$ & $5.90 E-01$ \\
\hline KEGG_PATHWAY & $\mathrm{T}$ cell receptor signaling pathway & 21 & 0.1 & $1.70 E-02$ & $3.20 E-01$ \\
\hline KEGG_PATHWAY & Endocytosis & 42 & 0.2 & $7.70 E-05$ & $4.70 E-03$ \\
\hline KEGG_PATHWAY & Axon guidance & 21 & 0.1 & $7.00 E-02$ & $6.40 E-01$ \\
\hline KEGG_PATHWAY & TGF-beta signaling pathway & 20 & 0.1 & $2.50 E-03$ & $7.40 E-02$ \\
\hline
\end{tabular}

Thresholds: count cut-off $\geq 20$; EASE $<0.1$. 
although screened by fold change $\geq 1.5$ and $P$ value $<0.05$, 23 miRNAs were significantly regulated by acupuncture treatment (Tables 1 and 2). These microarray data were further validated using qRT-PCR. The findings from this study suggest that miRNAs are involved in the therapeutic effects of acupuncture.

Our finding further showed that acupuncture treatment at specific acupoint elicits selective miRNA expression changes in SHRs (Figure 3). Acupoint specificity is the theoretical basis for meridian and acupuncture theory and is also the key factor for the application of effective and individualized acupuncture treatment [30, 31]. However, there is still some controversy about the existence of acupoint specificity [32-37]. Our data showed that acupuncture at taichong acupoint regulated miR-339, miR-223, and miR-145 expression, while acupuncture at nonacupoint failed to affect these miRNAs' expression. Other researches have observed acupoint-specific effect at taichong point in human brain by functional nuclear magnetic resonance imaging technique [38].

Our bioinformatics analysis showed that 23 miRNAs responded to acupuncture and these miRNAs might regulate 2963 targets. These miRNAs have many important physiological and pathological functions. miRNA-339, miR223, and miR-145 are highly conserved and have multiple targets predicted for them in both humans and rats [3941]. In addition, many studies have demonstrated that these miRNAs are involved in cell proliferation, apoptosis, and tumor suppressor. For example, miRNA-339 has been shown to have a central role in regulating the expression of intercellular cell adhesion molecule-1 [39]. Similarly, miR-223 has been found to negatively regulate progenitor proliferation and granulocyte differentiation and activation [42]. miR-145 has been proposed to inhibit human embryonic stem cell self-renewal, represses expression of pluripotency genes, and induces lineage-restricted differentiation [41]. miR-145 has also been proposed as a tumor suppressor that can target the $3^{\prime}$-untranslated region of the insulin receptor substrate-1 gene and dramatically inhibit the growth of colon cancer cells [43]. Therefore, acupuncture-responsive miRNAs may contribute to the therapeutic effects of acupuncture in hypertension through various pathways.

Our bioinformatic data also showed that there were 14 pathways regulated by the miRNAs responsive to acupuncture therapy (Table 3). Among these pathways, the neurotrophin signaling pathway plays critical roles in the regulation of brain activities and blood pressure control. Neurotrophins are a family of growth factors that induce the survival, development, and function of neurons [44, 45], which also play an important role in pathogenesis of hypertension [46] and in the neuroprotective activity of acupuncture treatment. The cardiovascular system is tightly controlled by the nervous system; neurons, cardiomyocytes, endothelial cells, and vascular smooth muscle cells are all well regulated by neurotrophins [46-49]. Our data suggest that acupuncture might regulate blood pressure through microRNAneurotrophin signaling pathway. Studies by us and others have demonstrated that acupuncture activated neurotrophin

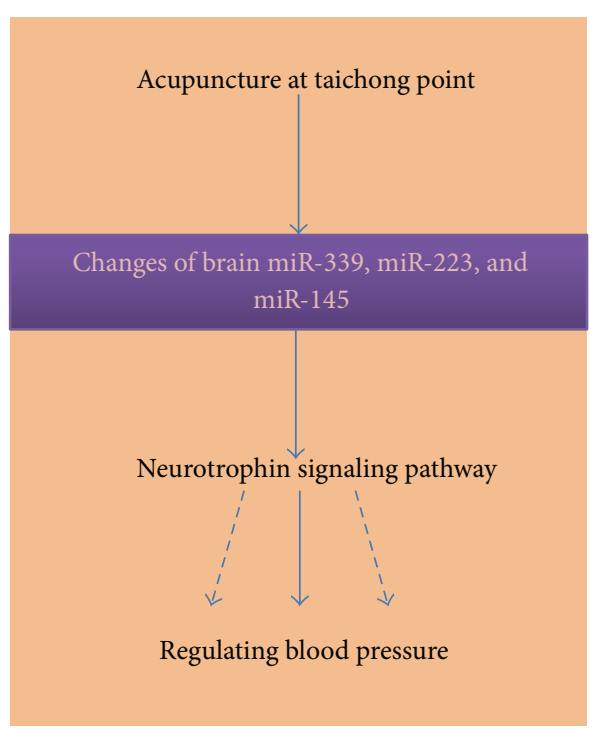

FIgUre 4: Proposed role of miRNAs in the antihypertensive effects of acupuncture.

signaling pathway and elicited neuroprotective activity [5052]. Recent studies have showed that microRNA can not only regulate neurotrophin expression, but also target their receptors and downstream signaling proteins $[53,54]$. For example, miR-206 was found to decrease brain-derived neurotrophic factor level $[53,54]$ and miR-21 regulated neuron growth factor signaling pathway in rats [55]. miR-592 was shown to modulate the induction of p75 neurotrophin receptor in neuronal ischemic injury [56]. Our data together with data from other groups suggest that acupuncture could modulate various miRNAs which consequently altered the function and activities of critical neuronal and cardiovascular factors contributing to the regulation of blood pressure (Figure 4). The acupuncture-responsive miRNAs are supposed to play important roles in the therapeutic effects of acupuncture treatment for hypertension, but further mechanistic studies are warranted to elicit how these responsive miRNAs contribute to the antihypertensive activity of acupuncture.

In addition, our bioinformatics data predicted that mitogen-activated protein kinase (MAPK) signaling pathway was possibly involved in the antihypertensive activity of acupuncture in rats. The MAPK signaling molecules have been recognized as important mediators in directing cellular responses to a diverse array of stimuli, such as proinflammatory cytokines and exposure to environmental compounds. They regulate gene expression, differentiation, apoptosis and many other cellular processes [57]. Studies have showed that MAPK signaling pathway was activated by acupuncture in the brain [58]. Further functional studies are needed to dissect the role of MAPK signalling pathway in the therapeutic effects of acupuncture.

In summary, our microarray study for the first time identified 222 differentially expressed miRNAs in the medulla of SHRs treated with acupuncture at the taichong acupoint. Among these miRNAs, 23 miRNAs were found to be differentially expressed in acupuncture-treated SHRs compared 
to untreated control rats. These 23 miRNAs could regulate 2963 target genes based on our bioinformatic analysis. Importantly, our RT-PCR assay has confirmed that miRNA-339, miR-223, and miR-145 were upregulated in SHRs treated with acupuncture at taichong acupoint in comparison with the nonacupoint group. Our findings have demonstrated significant changes of specific and selective miRNAs in rats when taichong acupoint was stimulated. Our data have revealed the specific miRNA profile changes in response to acupuncture treatment and strongly suggest that a selective panel of miRNAs play an important role in the antihypertensive activity of acupuncture therapy.

\section{Conflict of Interests}

The authors declare that there is no conflict of interests regarding the publication of this paper.

\section{Authors' Contribution}

Jia-You Wang and Hui Li equally contributed to this work as joint first authors.

\section{Acknowledgments}

The microarray experiments were performed by KangChen Bio-tech Inc., Shanghai, China. This project were supported by National Key Basic Research and Development Program (Grant no. 2012CB518504), Natural Science Foundation of Guangdong Province (Grant no. S2013010011547), National Natural Science Foundation of China (Grant no. 81173349), and University of South Florida College of Pharmacy Startup Fund.

\section{References}

[1] P. M. Kearney, M. Whelton, K. Reynolds, P. Muntner, P. K. Whelton, and J. He, "Global burden of hypertension: analysis of worldwide data," The Lancet, vol. 365, no. 9455, pp. 217-223, 2005.

[2] A. S. Go, D. Mozaffarian, V. L. Roger et al., "Heart disease and stroke statistics-2014 update a report from the American Heart Association," Circulation, vol. 129, no. 3, pp. e28-e292, 2014.

[3] A. H. Gradman, J. N. Basile, B. L. Carter, and G. L. Bakris, "Combination therapy in hypertension," Journal of the American Society of Hypertension, vol. 4, no. 1, pp. 42-50, 2010.

[4] A. Burke, D. M. Upchurch, C. Dye, and L. Chyu, "Acupuncture use in the United States: findings from the National Health Interview Survey," Journal of Alternative and Complementary Medicine, vol. 12, no. 7, pp. 639-648, 2006.

[5] P. M. Barnes, B. Bloom, R. L. Nahin, and N. C. f. H. Statistics, Complementary and Alternative Medicine Use among Adults and Children: United States, 2007, Department of Health and Human Services, Centers for Disease Control and Prevention, National Center for Health Statistics, Hyattsville, MD, USA, 2008.

[6] NIN Consensus Development Panel on Acupuncture, “Acupuncture," JAMA, vol. 280, no. 17, pp. 1518-1524, 1998.
[7] K. C. Tam and H. H. Yiu, "The effect of acupuncture on essential hypertension," The American Journal of Chinese Medicine, vol. 3, no. 4, pp. 369-375, 1975.

[8] Y. J. Chiu, A. Chi, and I. A. Reid, "Cardiovascular and endocrine effects of acupuncture in hypertensive patients," Clinical and Experimental Hypertension, vol. 19, no. 7, pp. 1047-1063, 1997.

[9] C. Yin, B. Seo, H.-J. Park et al., "Acupuncture, a promising adjunctive therapy for essential hypertension: a double-blind, randomized, controlled trial," Neurological Research, vol. 29, no. 1, pp. S98-S103, 2007.

[10] H. Chen, J. Dai, X. Zhang et al., "Hypothalamus-related resting brain network underlying short-term acupuncture treatment in primary hypertension," Evidence-Based Complementary and Alternative Medicine, vol. 2013, Article ID 808971, 9 pages, 2013.

[11] D. D. Kim, A. M. Pica, R. G. Durán, and W. N. Durán, "Acupuncture reduces experimental renovascular hypertension through mechanisms involving nitric oxide synthases," Microcirculation, vol. 13, no. 7, pp. 577-585, 2006.

[12] W. Zhou and J. C. Longhurst, "Neuroendocrine mechanisms of acupuncture in the treatment of hypertension," Evidence-Based Complementary and Alternative Medicine, vol. 2012, Article ID 878673, 9 pages, 2012.

[13] I. V. Anshelevich, M. A. Merson, and G. A. Afanas'eva, "Serum aldosterone level in patients with hypertension during treatment by acupuncture," Terapevticheskii Arkhiv, vol. 57, no. 10, pp. 42-45, 1984.

[14] C. Yuefeng, Q. Hong, and L. Lan, "Effects of acupuncture on contents of plasma endothelin and angiotensin II in the patient of hypertension," Chinese Acuponcture Moxibustion, vol. 11, no. 29,2000

[15] P. Li, S. C. Tjen-A-Looi, and J. C. Longhurst, "Acupuncture's role in cardiovascular homeostasis," in Current Research in Acupuncture, pp. 457-486, Springer, New York, NY, USA, 2013.

[16] W. Zhou, S. C. Tjen-A-Looi, and J. C. Longhurst, "Brain stem mechanisms underlying acupuncture modality-related modulation of cardiovascular responses in rats," Journal of Applied Physiology, vol. 99, no. 3, pp. 851-860, 2005.

[17] D. P. Bartel, "MicroRNAs: genomics, biogenesis, mechanism, and function," Cell, vol. 116, no. 2, pp. 281-297, 2004.

[18] V. Ambros, “The functions of animal microRNAs," Nature, vol. 431, no. 7006, pp. 350-355, 2004.

[19] P. Coyle, "Different susceptibilities to cerebral infarction in spontaneously hypertensive (SHR) and normotensive SpragueDawley rats," Stroke, vol. 17, no. 3, pp. 520-525, 1986.

[20] F. Hopp, C. Dean, and J. Seagard, "Endocannabinoid effects on neuronal function in the NTS of hypertensive rats (1130.3)," The FASEB Journal, vol. 28, no. 1, supplement, pp. 1130-1133, 2014.

[21] M. Tsai and Y. Wang, "Effect of laser stimulation of acupoint taichong (LR3) on blood pressure and heart rate variability," The Internet Journal of Alternative Medicine, vol. 9, no. 1, 2014.

[22] X. Lai, J. Wang, N. R. Nabar et al., "Proteomic response to acupuncture treatment in spontaneously hypertensive rats," PLoS ONE, vol. 7, no. 9, Article ID e44216, 2012.

[23] G. Litscher, W.-P. Cheng, G.-Y. Cheng et al., "Acupuncture point laterality: investigation of acute effects of Quchi (LI11) in patients with hypertension using heart rate variability," Evidence-Based Complementary and Alternative Medicine, vol. 2014, Article ID 979067, 6 pages, 2014.

[24] J. Wang, C. Tang, Z. He et al., "Effect of moderate acupuncturestimulation of "Taichong" (LR 3) on blood pressure and plasma endothelin-1 levels in spontaneous hypertension rats," Zhen $C i$ Yan Jiu, vol. 36, no. 1, pp. 36-39, 2011. 
[25] W. L. Chilton, F. Z. Marques, J. West et al., "Acute exercise leads to regulation of telomere-associated genes and microRNA expression in immune cells," PloS One, vol. 9, no. 4, Article ID e92088, 2014.

[26] F. C. Mooren, J. Viereck, K. Krüger, and T. Thum, "Circulating microRNAs as potential biomarkers of aerobic exercise capacity," American Journal of Physiology, Heart and Circulatory Physiology, vol. 306, no. 4, pp. H557-H563, 2013.

[27] A. L. Baggish, A. Hale, R. B. Weiner et al., "Dynamic regulation of circulating microRNA during acute exhaustive exercise and sustained aerobic exercise training," The Journal of Physiology, vol. 589, no. 16, pp. 3983-3994, 2011.

[28] W. Aoi, Y. Naito, K. Mizushima et al., "The microRNA miR696 regulates PGC- $1 \alpha$ in mouse skeletal muscle in response to physical activity," The American Journal of PhysiologyEndocrinology and Metabolism, vol. 298, no. 4, pp. E799-E806, 2010.

[29] M. Cosín-Tomás, M. J. Alvarez-López, S. Sanchez-Roige et al., "Epigenetic alterations in hippocampus of SAMP8 senescent mice and modulation by voluntary physical exercise," Frontiers in Aging Neuroscience, vol. 6, article 51, 2014.

[30] B. Pearson, "Progress of research on specificity of meridian acupoint efficacy," Chinese Journal of Integrative Medicine, vol. 19, no. 12, pp. 889-893, 2013.

[31] J.-J. Xing, B.-Y. Zeng, J. Li, Y. Zhuang, and F.-R. Liang, "Acupuncture point specificity," International Review of Neurobiology, vol. 111, pp. 49-65, 2013.

[32] L. Chen, J. Tang, P. F. White et al., "The effect of location of transcutaneous electrical nerve stimulation on postoperative opioid analgesic requirement: acupoint versus nonacupoint stimulation," Anesthesia and Analgesia, vol. 87, no. 5, pp. 11291134, 1998.

[33] J. L. Fang, T. Krings, J. Weidemann, I. G. Meister, and A. Thron, "Functional MRI in healthy subjects during acupuncture: different effects of needle rotation in real and false acupoints," Neuroradiology, vol. 46, no. 5, pp. 359-362, 2004.

[34] D. Li, M. Yang, L. Zhao et al., "Acupuncture for chronic, stable angina pectoris and an investigation of the characteristics of acupoint specificity: study protocol for a multicenter randomized controlled trial," Trials, vol. 15, no. 1, p. 50, 2014.

[35] B. J. Na, G. H. Jahng, S. U. Park et al., "An fMRI study of neuronal specificity of an acupoint: electroacupuncture stimulation of Yanglingquan (GB34) and its sham point," Neuroscience Letters, vol. 464, no. 1, pp. 1-5, 2009.

[36] L. Li, W. Qin, L. Bai, and J. Tian, "Exploring vision-related acupuncture point specificity with multivoxel pattern analysis," Magnetic Resonance Imaging, vol. 28, no. 3, pp. 380-387, 2010.

[37] J. Yang, F. Zeng, Y. Feng et al., "A PET-CT study on the specificity of acupoints through acupuncture treatment in migraine patients," BMC Complementary and Alternative Medicine, vol. 12, no. 1, article 123, 2012.

[38] B. Yan, K. Li, J. Xu et al., "Acupoint-specific fMRI patterns in human brain," Neuroscience Letters, vol. 383, no. 3, pp. 236-240, 2005.

[39] R. Ueda, G. Kohanbash, K. Sasaki et al., "Dicer-regulated microRNAs 222 and 339 promote resistance of cancer cells to cytotoxic T-lymphocytes by down-regulation of ICAM-1," Proceedings of the National Academy of Sciences of the United States of America, vol. 106, no. 26, pp. 10746-10751, 2009.

[40] F. Fazi, A. Rosa, A. Fatica et al., "A minicircuitry comprised of microRNA-223 and transcription factors NFI-A and C/EBP $\alpha$ regulates human granulopoiesis," Cell, vol. 123, no. 5, pp. 819831, 2005.

[41] J. B. Johnnidis, M. H. Harris, R. T. Wheeler et al., "Regulation of progenitor cell proliferation and granulocyte function by microRNA-223," Nature, vol. 451, no. 7182, pp. 1125-1129, 2008.

[42] N. Xu, T. Papagiannakopoulos, G. Pan, J. A. Thomson, and K. S. Kosik, "MicroRNA-145 regulates OCT4, SOX2, and KLF4 and represses pluripotency in human embryonic stem cells," Cell, vol. 137, no. 4, pp. 647-658, 2009.

[43] G. la Rocca, M. Badin, B. Shi et al., "Mechanism of growth inhibition by microRNA 145: the role of the IGF-I receptor signaling pathway," Journal of Cellular Physiology, vol. 220, no. 2, pp. 485-491, 2009.

[44] B. L. Hempstead, "Dissecting the diverse actions of pro- and mature neurotrophins," Current Alzheimer Research, vol. 3, no. 1, pp. 19-24, 2006.

[45] L. F. Reichardt, "Neurotrophin-regulated signalling pathways," Philosophical Transactions of the Royal Society B: Biological Sciences, vol. 361, no. 1473, pp. 1545-1564, 2006.

[46] C. Emanueli, M. Meloni, W. Hasan, and B. A. Habecker, "The biology of neurotrophins: cardiovascular function," in Neurotrophic Factors, pp. 309-328, Springer, 2014.

[47] S.-H. Zhang and R. A. Rush, "Neurotrophin 3 is increased in the spontaneously hypertensive rat," Journal of Hypertension, vol. 19, no. 12, pp. 2251-2256, 2001.

[48] C. G. Clark, D. D. Kline, D. L. Kunze, D. M. Katz, and E. M. Hasser, "Brain derived neurotrophic factor (BDNF) in the nucleus tractus solitarii (nTS) contributes to sympathoexcitation in spontaneously hypertensive rats," The FASEB Journal, vol. 25, pp. 841-843, 2011.

[49] A. Vermehren-Schmaedick, V. K. Jenkins, H. Hsieh et al., "Upregulation of brain-derived neurotrophic factor expression in nodose ganglia and the lower brainstem of hypertensive rats," Journal of Neuroscience Research, vol. 91, no. 2, pp. 220-229, 2013.

[50] D. Lin, I. De La Pena, L. Lin et al., “The neuroprotective role of acupuncture and activation of the BDNF signaling pathway," International Journal of Molecular Sciences, vol. 15, no. 2, pp. 3234-3252, 2014.

[51] L. Manni, M. Albanesi, M. Guaragna, S. Barbaro Paparo, and L. Aloe, "Neurotrophins and acupuncture," Autonomic Neuroscience: Basic and Clinical, vol. 157, no. 1-2, pp. 9-17, 2010.

[52] M. Soligo, S. L. Nori, V. Protto, F. Florenzano, and L. Manni, "Acupuncture and neurotrophin modulation," International Review of Neurobiology, vol. 111, no. 91, 2013.

[53] N. Tian, Z. Cao, and Y. Zhang, "MiR-206 decreases brainderived neurotrophic factor levels in a transgenic mouse model of Alzheimer's disease," Neuroscience Bulletin, vol. 30, no. 2, pp. 191-197, 2014.

[54] S. T. Lee, K. Chu, K. H. Jung et al., "miR-206 regulates brain-derived neurotrophic factor in Alzheimer disease model," Annals of Neurology, vol. 72, no. 2, pp. 269-277, 2012.

[55] E. Montalban, N. Mattugini, and R. Ciarapica, "MiR-21 is an Ngf-modulated MicroRNA that supports Ngf signaling and regulates neuronal degeneration in PC12 cells," Neuromolecular Medicine, vol. 16, no. 2, pp. 415-430, 2014.

[56] K. Irmady, K. A. Jackman, V. A. Padow et al., "MiR-592 regulates the induction and cell death-promoting activity of p75NTR in neuronal ischemic injury," The Journal of Neuroscience, vol. 34, no. 9, pp. 3419-3428, 2014. 
[57] G. Pearson, F. Robinson, T. B. Gibson et al., "Mitogen-activated protein (MAP) kinase pathways: regulation and physiological functions," Endocrine Reviews, vol. 22, no. 2, pp. 153-183, 2001.

[58] Y.-H. Gao, S.-P. Chen, J.-Y. Wang et al., "Differential proteomics analysis of the analgesic effect of electroacupuncture intervention in the hippocampus following neuropathic pain in rats," BMC Complementary and Alternative Medicine, vol. 12, no. 1, article 241, 2012. 


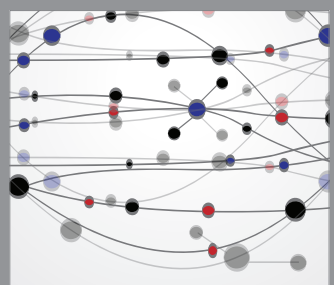

The Scientific World Journal
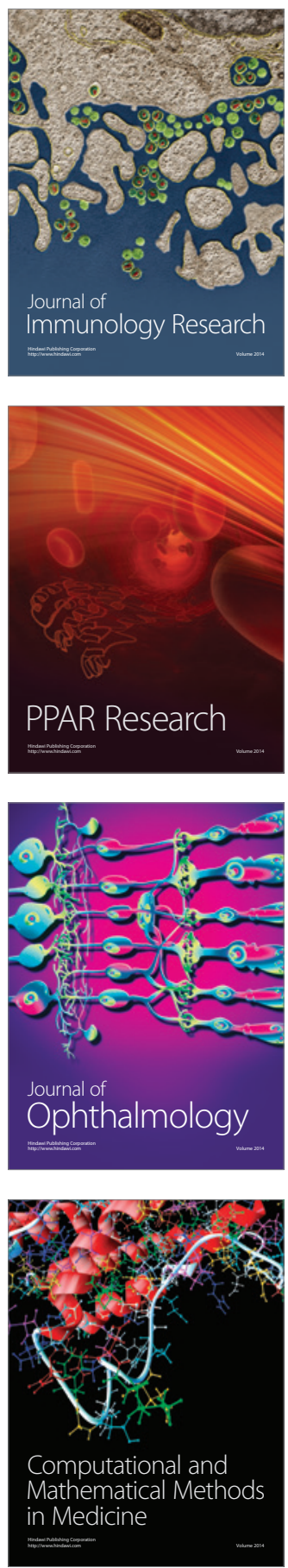

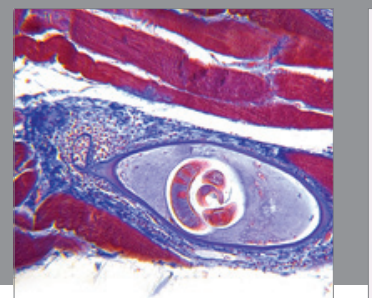

Gastroenterology

Research and Practice
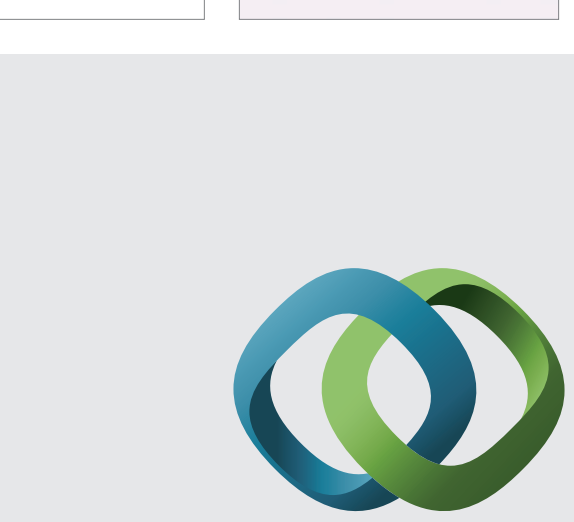

\section{Hindawi}

Submit your manuscripts at

http://www.hindawi.com
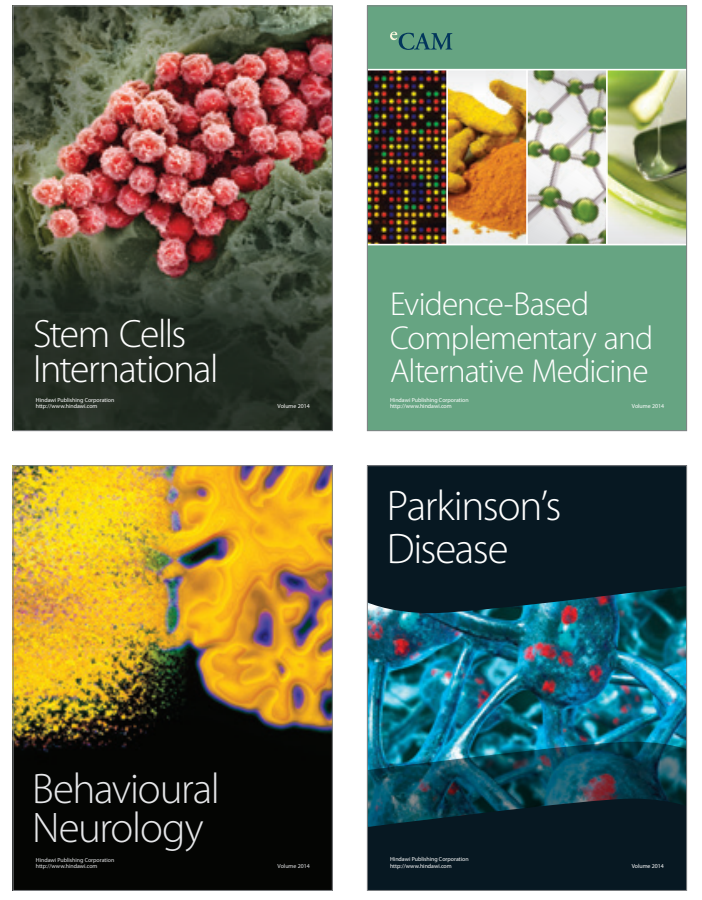
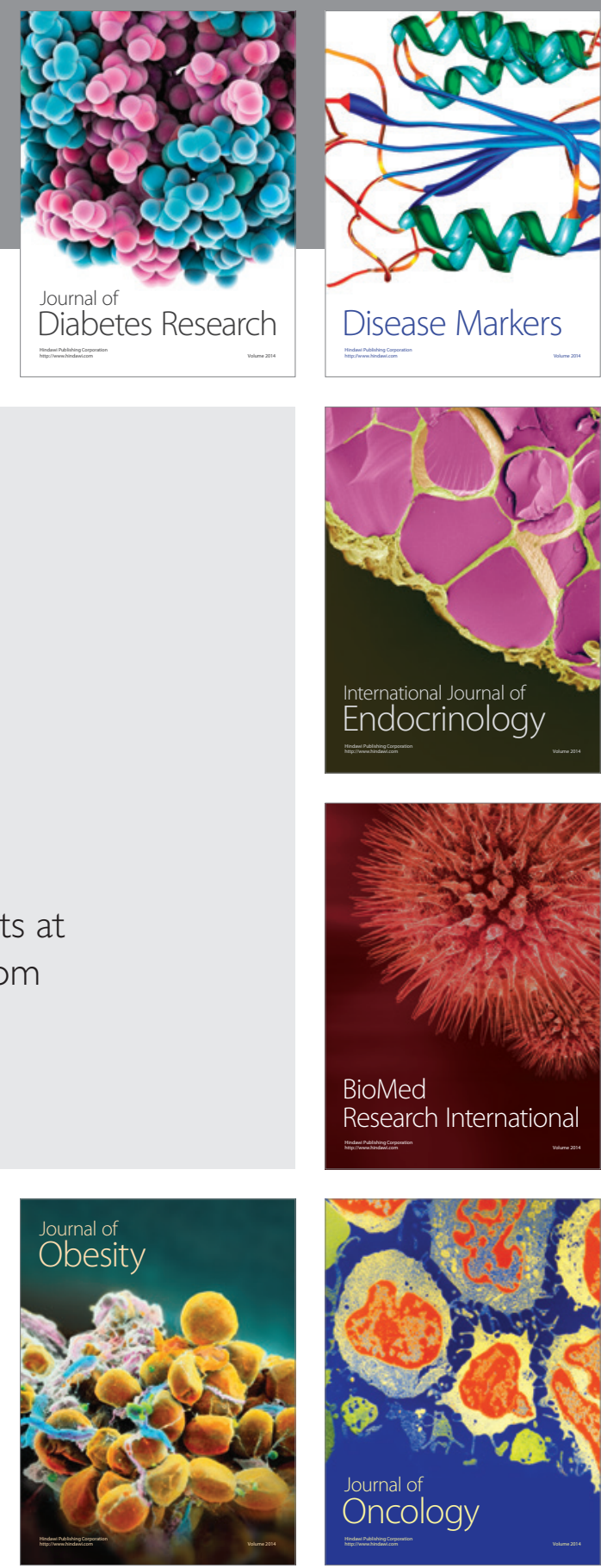

Disease Markers
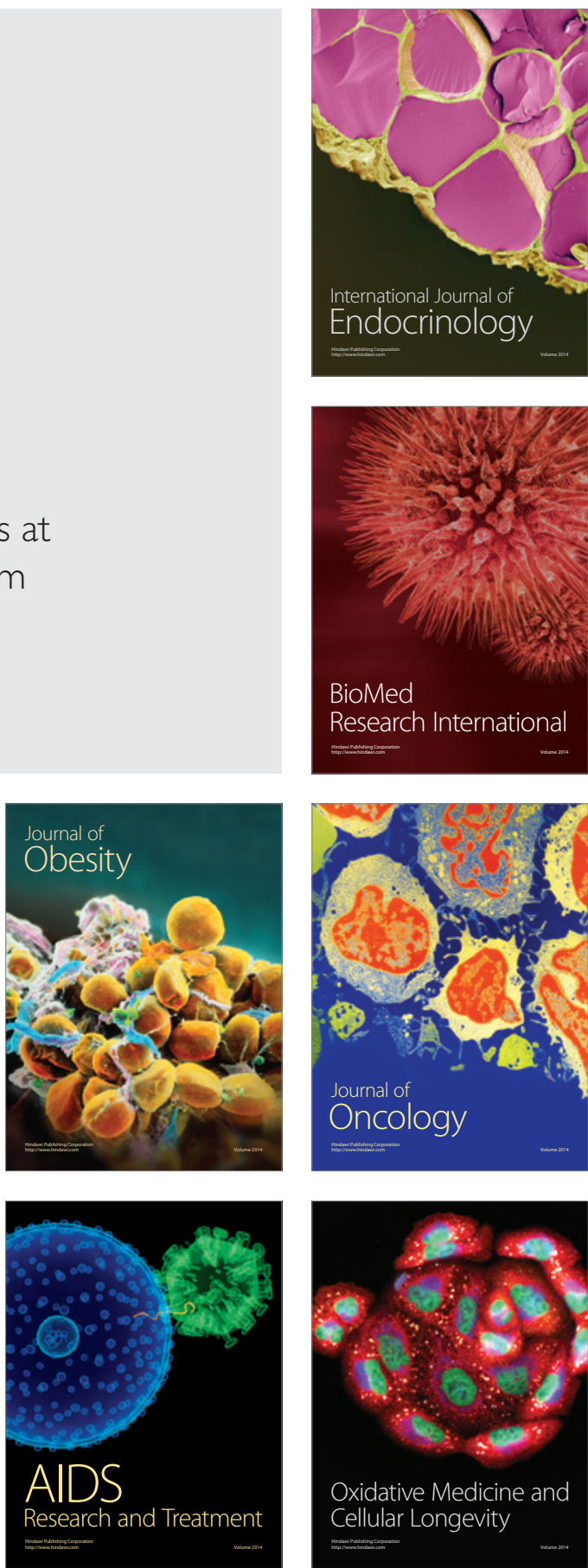